\title{
Aplicación Sigma y Grupos Facebook: Evaluación de la Usabilidad y Aceptación Tecnológica por Estudiantes de Ingeniería de Sistemas de la Universidad Técnica de Ambato en Ecuador
}

\author{
Érika Reyes-Garcés, Félix Fernández-Peña*, Wilson Pérez-Nata y Pilar Urrutia-Urrutia \\ Facultad de Ingeniería en Sistemas, Electrónica e Industrial, Universidad Técnica de Ambato. Av. Los \\ Chasquis y Río Payamino. Ambato, Ecuador (e-mail: ereyes5148@uta.edu.ec; fo.fernandez@uta.edu.ec; \\ wg.perez@uta.edu.ec; elsapurrutia@uta.edu.ec).
}

* Autor a quien debe ser dirigida la correspondencia

Recibido Mar. 7, 2018; Aceptado May. 16, 2018; Versión final Jun. 11, 2018, Publicado Oct. 2018

\begin{abstract}
Resumen
En este trabajo se compara el uso de un grupo Facebook con el de una aplicación Facebook, como posible alternativa para solucionar las dificultades previamente encontradas en el uso de esta red social en un proceso de aprendizaje colaborativo. Se trata de una investigación de tipo aplicada, cuantitativa y exploratoria que siguió una metodología de investigación experimental. El estudio se basó en el Modelo de Aceptación Tecnológica y evaluación heurística. Como resultado, se comprobó la flexibilidad de la plataforma Facebook y el nivel de aceptación que tienen los grupos Facebook entre los estudiantes de nivel intermedio de Ingeniería en Sistemas de la Universidad Técnica de Ambato. Se concluye que fue posible personalizar el aprendizaje colaborativo en Facebook con la construcción de la aplicación Sigma. Si embargo, contrario a lo esperado, el uso de un grupo Facebook fue aceptado por los estudiantes que participaron en el experimento como la mejor alternativa para el uso de esta plataforma en el aprendizaje colaborativo.
\end{abstract}

\section{App Sigma and Facebook Groups: Evaluation of the Usability and Technology Acceptance by Software Engineering Students of Universidad Técnica de Ambato in Ecuador}

\begin{abstract}
In this paper, the use of a Facebook group is compared with a Facebook app, as a viable alternative to overcome the difficulties previously found when using this social network in a collaborative learning process. This is an applied and quantitative and exploratory research carried out using the experiment methodology. This study is based on the Technology Acceptance Model and the heuristic evaluation. The flexibility of the Facebook platform was verified, as well as the acceptance of Facebook groups among sophomores studying software engineering at the Universidad Técnica de Ambato in Ecuador. It is concluded that it was possible to personalize collaborative learning in Facebook with the construction of Sigma. However, contrary to expectations, using a Facebook group is considered by those students who participated in the experiment as the best alternative for using this platform in collaborative learning.
\end{abstract}

Keywords: usability; social media; collaborative learning; Facebook platform; technology acceptance model 


\section{INTRODUCCIÓN}

El aprendizaje colaborativo se da cuando el conocimiento se genera a partir de la interacción de miembros de una población que comparten experiencias (McCarthy, 2015). El uso de Facebook ha sido ampliamente estudiado sin llegar a un consenso entre defensores de su uso en el ámbito del aprendizaje colaborativo (Manca et al., 2013; Castro y González-Palta, 2016; Dohn, 2017; Chang et al., 2017; Moghavvemi et al., 2017; Rap y Blonder, 2017; Guo et al., 2018) y detractores (Çoklar, 2012; Kirschner, 2015; Tsovaltzi et al., 2017; Thompson, 2017; Lin, 2018). No obstante, actualmente se reconoce 1) la amplia aceptación del uso de esta red social entre los estudiantes universitarios (Dohn, 2017; Moghavvemi et al., 2017; Lin, 2018) y 2) la importancia de que los facilitadores del proceso de aprendizaje aprovechen las bondades que esta plataforma tecnológica pueda ofrecerles (Guo et al., 2018).

Desde hace varios años se reporta el uso que ha tenido esta plataforma para compartir recursos de información entre personas y organizaciones. Manca et al. (2013) identificaron cinco usos educativos de Facebook, dígase: soporte y ayuda en el aprendizaje colaborativo de los estudiantes, desarrollo colaborativo de contenidos, compartir recursos educativos, entrega de contenidos extracurriculares y apoyo en el autoaprendizaje. De La Hoz et al. (2015) comprobaron el uso aceptable que tuvo Facebook como herramienta educativa en la Universidad Antonio Nariño, sede Cartagena. Chang et al. (2017) y Dohn (2017) documentaron casos de éxito actuales del uso de Facebook en sistemas de aprendizaje colaborativo en el área de la salud y ciencias biológicas. Por su parte, Rap y Blonder (2017), después de un estudio longitudinal de dos años de duración, concluyeron que Facebook es una herramienta válida para facilitar la supervisión del aprendizaje, tras haber encontrado una correlación positiva entre actividades de aprendizaje de Química y la actitud de los estudiantes hacia el uso de Facebook en este contexto.

Estos resultados tienen mayor relevancia al considerar el impacto de Facebook en el aprendizaje móvil para el que los estudiantes universitarios han mostrado predisposición según el estudio de Lagunes et al. (2017). No obstante, si bien se destaca la capacidad de las redes sociales para incentivar el desarrollo de una conciencia de la competencia cultural y mantener el interés en los estudiantes (Chang et al. 2017), Dohn resalta que también crea barreras en la comunicación, las que se deben a distracciones, asuntos éticos y a cierta depreciación de las actividades derivadas de la cotidianidad en Facebook (Dohn, 2017).

Del mismo modo, el estudio cualitativo de la influencia de Facebook en procesos de aprendizaje colaborativo de Pimmer et al. (2017) concluye que entre los estudiantes y facilitadores del proceso de aprendizaje surgen tensiones en el intento de lograr el aprendizaje y la supervisión en los espacios de la red social. Por su parte, Castro y González-Palta (2016), Moghavvemi et al. (2017) y Guo et al. (2018) defienden el uso de Facebook por su impacto en el desarrollo de una capacidad crítica en los estudiantes, la percepción positiva que tienen los estudiantes sobre el uso de Facebook en la educación y por su influencia positiva en la capacidad de comunicación estudiante - instructor, respectivamente.

Otros autores señalan que Facebook no puede ser considerado como un entorno de aprendizaje, ya que fue desarrollado para la interacción social informal (Kirschner et al., 2015). Çoklar (2012) ha mencionado que, en el ámbito educativo, Facebook enfrenta 1) los inconvenientes de estar ligado al entretenimiento, 2) los problemas de los mecanismos de control y 3 ) una excesiva convergencia de información. En el estudio de Lin (2018), se reflexiona sobre los beneficios pedagógicos que tiene el anonimato que no existe en las redes sociales, cuando se trata de determinados escenarios de aprendizaje colaborativo y fundamenta el resultado de su investigación a través del análisis de cómo la falta de anonimato afectó la usabilidad percibida y la actitud hacia el uso de los participantes en el experimento llevado a cabo.

Un estudio de caso llevado a cabo por Junco (2015) evidencia que es en los primeros años de estudios universitarios donde el rendimiento académico se podría estar viendo afectado negativamente por el uso de Facebook y no en años superiores, aunque, de cualquier manera, la afectación potencial está latente. Posteriormente, Thompson (2017) analizó nuevamente la correlación negativa que se presenta entre la frecuencia de uso de las tecnologías de comunicación, tales como las redes sociales, y el desempeño académico, en un estudio que involucró la participación de 6000 estudiantes en los primeros años de la universidad, corroborando los resultados de Junco (2015) para estudiantes de niveles iniciales. Por su parte, Tsovaltzi et al. (2017) valoran como positiva la capacidad de argumentación que se desarrolla a través de Facebook, pero concluyen que no se logra una consolidación del conocimiento individual en los estudiantes.

En la literatura consultada, hemos identificado tres opciones que brinda Facebook para personalizar el uso de la red social en un contexto específico: página Facebook, ampliamente utilizada en campañas de mercadotecnia (Zorkociova y Vankova, 2016), grupo Facebook, que se ha utilizado fundamentalmente para mejorar la comunicación estudiante-profesor (Guo et al., 2018) y aplicación Facebook, que permite explotar 
las capacidades interactivas de la plataforma en las más diversas áreas del conocimiento (Li et al., 2018). Estas tres alternativas (caracterizadas en la tabla 1) tienen como base común la plataforma de la red social, la que registra las interacciones sociales (compartir y me gusta) que tienen lugar sobre los recursos de información que se comparten. El uso de una página Facebook se descarta en el ámbito académico puesto que sus prestaciones no son las requeridas en cuanto al nivel de interactividad que los usuarios registrados pueden tener, entre ellos, y con el contenido que se publica.

Tabla 1: Descripción de las opciones de personalización de Facebook.

\begin{tabular}{|c|c|c|c|}
\hline \multirow{2}{*}{ Características } & \multicolumn{3}{|c|}{ Opciones Facebook } \\
\hline & Página Facebook & Grupo Facebook & Aplicación Facebook \\
\hline Interfaz & $\begin{array}{l}\text { Personalizada (con } \\
\text { limitaciones) por el usuario } \\
\text { dueño de la página. }\end{array}$ & Definida por Facebook. & $\begin{array}{l}\text { Definida por el programador y, } \\
\text { si este lo decide, también por } \\
\text { el usuario. }\end{array}$ \\
\hline $\begin{array}{l}\text { Lenguaje de } \\
\text { programación }\end{array}$ & HTML + CSS & - & $\begin{array}{l}\text { El seleccionado por el } \\
\text { desarrollador de la aplicación, } \\
\text { además de HTML y CSS. }\end{array}$ \\
\hline $\begin{array}{l}\text { Tipo de } \\
\text { Contenido }\end{array}$ & $\begin{array}{l}\text { El que decida el editor de la } \\
\text { página además de } \\
\text { comentarios y acciones } \\
\text { sociales de otros usuarios } \\
\text { de esta. }\end{array}$ & $\begin{array}{l}\text { Publicación secuencial } \\
\text { de mensajes, } \\
\text { conversaciones, } \\
\text { documentos, audios y } \\
\text { videos. }\end{array}$ & El que decida el programador. \\
\hline $\begin{array}{l}\text { Presentación } \\
\text { de información }\end{array}$ & $\begin{array}{l}\text { Determinada por el creador } \\
\text { de la página. }\end{array}$ & $\begin{array}{l}\text { Determinada por } \\
\text { Facebook. }\end{array}$ & $\begin{array}{l}\text { Determinada por el } \\
\text { desarrollador de la aplicación. }\end{array}$ \\
\hline
\end{tabular}

Teniendo en cuenta las dos alternativas que restan, la pregunta de investigación que nos propusimos responder es: ¿el desarrollo de una aplicación Facebook para el aprendizaje colaborativo incrementa la aceptación de la tecnología y usabilidad de la plataforma Facebook en el ámbito educativo con relación al uso de un grupo Facebook? El objetivo de este artículo es discutir los resultados obtenidos en la evaluación cuantitativa de la usabilidad de grupos Facebook y de la alternativa que representa el uso de Sigma, una aplicación Facebook que implementamos para personalizar la red social para el proceso de aprendizaje.

\section{METODOLOGÍA}

La ejecución de la presente investigación se dividió en tres fases: 1) construcción de una aplicación Facebook concebida explícitamente para apoyar el aprendizaje colaborativo en esta red social, 2) determinar qué método de evaluación utilizar para comparar la aplicación Facebook con el uso de un grupo Facebook y 3) ejecutar un experimento diseñado para evaluar cuantitativamente el éxito de Sigma con relación al uso de un grupo Facebook.

\section{Construcción de la aplicación Facebook.}

El diseño de Sigma tuvo en cuenta las desventajas del uso de Facebook en el ámbito educativo que fueron identificadas por Çoklar (2012), y que han sido referidas en la introducción a este trabajo. Se pretende que el resultado sea la construcción de un repositorio de información útil en el proceso de aprendizaje colaborativo, desligado del entretenimiento alrededor del uso de la red social. Se diseñó que Sigma tuviera opciones como: 1) clasificar los recursos compartidos por categorías de interés en la temática estudiada, 2) ordenar, además de por fecha de publicación (como ocurre en el grupo Facebook), por cantidad de interacciones sociales y 3 ) filtrar las publicaciones por categoría de interés. Estas opciones están concebidas para evitar una excesiva convergencia de información.

En cuanto a mecanismos de control, los facilitadores quedan en condiciones de monitorear y evaluar la calidad del contenido que se publica. Para facilitarlo, a diferencia del grupo Facebook, se concibió que los usuarios de Sigma pudieran jugar tres roles fundamentales: 1) quienes tienen acceso a recursos de información que se publican por otros usuarios e interactúan socialmente en la red social alrededor de determinado recurso publicado con compartir y me gusta, 2) quienes, además de esto, publican recursos de información y 3) quienes publican recursos de información, comparten su punto de vista a través de sus publicaciones, pero no hacen compartir o me gusta sobre los recursos de información publicados. El acceso a la aplicación es a través de Facebook, con excepción de quienes juegan el tercer rol mencionado, en cuyo caso acceden al aplicativo directamente a través del sitio web de la universidad. Facilitadores y estudiantes participan del proceso de aprendizaje colaborativo y se respeta su decisión de formar parte, o no, de la red social Facebook. 


\section{Método de Evaluación de Resultados}

Para definir el método de evaluación de resultados, primeramente, se llevó a cabo una búsqueda en la base de datos de Scopus utilizando las palabras claves "Facebook", "usability" y "evaluation" entre los años 2013 y 2017. Del total de 61 trabajos encontrados, siete de ellos fueron seleccionados por su relación con la investigación propuesta. El análisis de estos artículos permitió identificar que los métodos de evaluación que se aplicaron fueron la evaluación heurística (McCarthy, 2015; Li et al., 2016; Park et al., 2016) y TAM (Rauniar et al., 2014; Moghavvemi et al., 2017; Chintalapati y Daruri, 2017; Guo et al., 2018).

La evaluación heurística tiene como función encontrar los problemas en el diseño de aplicativos, con el objetivo de corregir los problemas encontrados en futuras versiones del software (McCarthy, 2015; Park et al., 2016). Los aspectos que se miden incluyen: diseño de interfaz, lenguaje y ayuda al usuario (Li et al., 2016; Hammouri y Abu-Shanab, 2018). TAM define como criterios de medida la facilidad de uso percibida (PEoU, del inglés perceived ease of use) y la usabilidad percibida (PU, del inglés perceived usefulness). PEoU se entiende como el grado de esfuerzo que una persona cree que necesita para el manejo de un aplicativo informático y PU como el grado en que una persona cree que hace uso de una tecnología para mejorar su trabajo. Los criterios mencionados se complementan con la medida de la actitud hacia el uso ( $\mathrm{A}$, del inglés attitude toward using), la intención de uso (BI, del inglés behavorial intention to use) y el uso real del aplicativo.

Finalmente, se decidió que la evaluación de los resultados del estudio experimental a llevar a cabo estuviera basada en el Modelo de Aceptación de Tecnología (TAM) y la evaluación heurística, ampliamente utilizados actualmente en estudios de aceptación de tecnología en el ámbito educativo (Park et al., 2016; Moghavvemi et al., 2017; Chintalapati y Daruri, 2017; Hammouri y Abu-Shanab, 2018; Scherer et al., 2018).

\section{Diseño del Experimento}

La hipótesis nula quedó planteada como: La aplicación Sigma incrementará la aceptación de la tecnología y usabilidad de la plataforma Facebook en el ámbito educativo con respecto al uso de un grupo Facebook. Para comprobarla, se diseñó una unidad de aprendizaje colaborativo en la materia Estructuras de Datos referente al contenido de Árboles y Grafos. La materia se seleccionó teniendo en cuenta 1) que el aprendizaje colaborativo fuera relevante para cumplir los objetivos de formación perseguidos y 2) que fuera una materia de la especialidad. Se desplegó entonces una instancia de Sigma y un grupo Facebook con nombre "Árboles y Grafos". La figura 1 muestra una vista parcial de la apariencia del grupo Facebook.

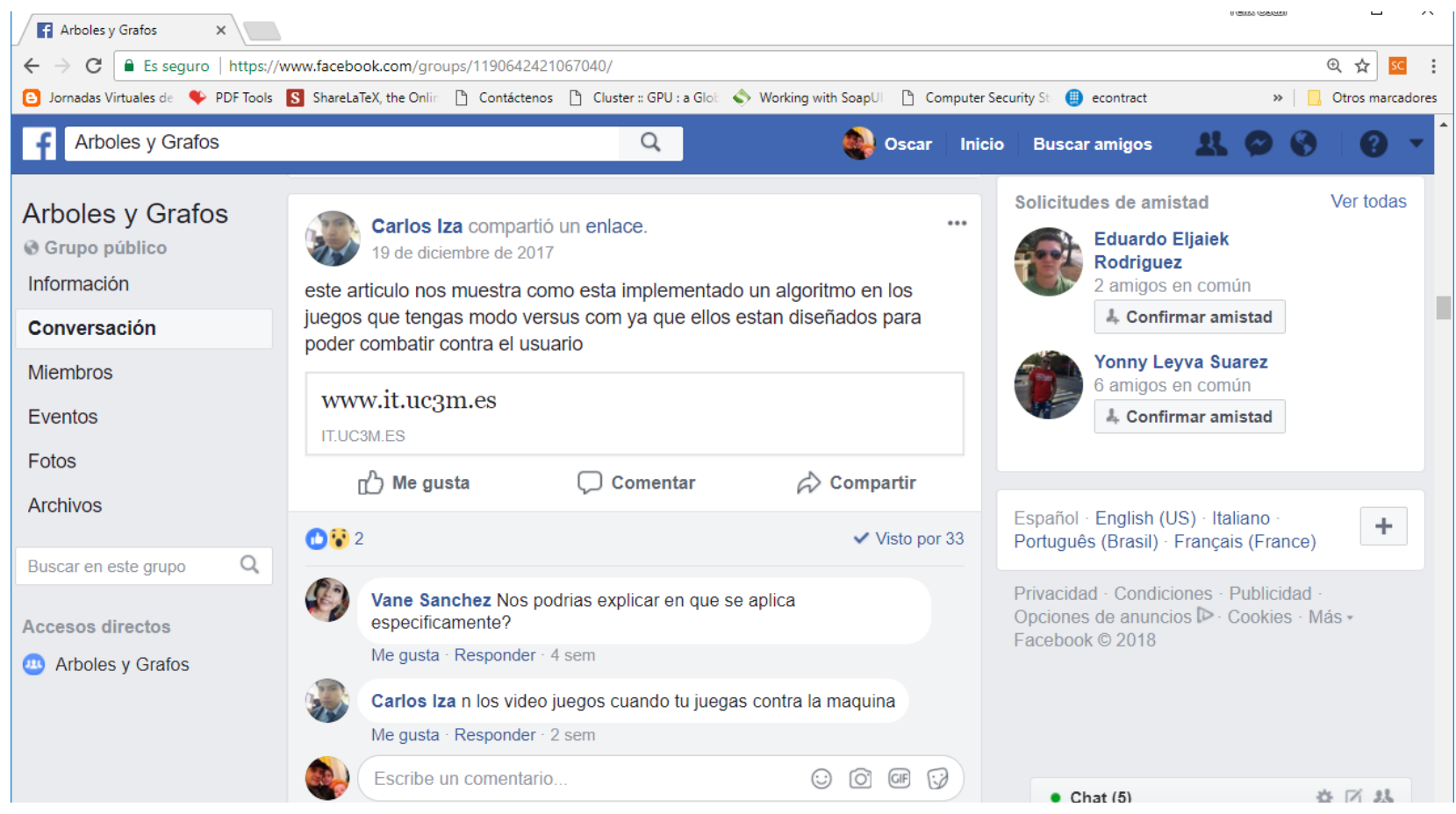

Fig. 1: Apariencia del grupo Facebook Árboles y Grafos.

Sigma y el grupo Facebook se utilizaron por parte de los estudiantes con el mismo objetivo: aprender, de forma colaborativa. Estas herramientas permitieron compartir recursos disponibles en Internet, dialogar con relación al impacto de las áreas de aplicación de árboles y grafos e interactuar socialmente con relación al impacto de los materiales compartidos utilizando las opciones me gusta y compartir. 
Al igual que en el estudio de grupos Facebook de Castro y González-Palta (2016), y en el de Po-Hsun y Li-Wei (2018), sobre la eficacia del aprendizaje en grupo, los estudiantes se agruparon aleatoriamente en dos grupos. Un grupo trabajó con Sigma (grupo experimental) y otro con el grupo Facebook (grupo control). Durante el proceso de enseñanza-aprendizaje, los estudiantes construyeron un repositorio compartido de recursos referentes a la aplicación de árboles y grafos en la ingeniería. El contenido de los recursos compartidos se discutió entre los participantes en el curso para facilitar el proceso de aprendizaje del uso práctico de estas estructuras de datos. Al concluir el proceso de aprendizaje colaborativo se aplicó una encuesta que permitió cuantificar el nivel de aceptación de la tecnología y el nivel de usabilidad, tanto del grupo Facebook como de Sigma.

La encuesta incluyó 22 ítems. En una primera sección (de cinco ítems) se recuperó información demográfica de los participantes en el estudio. Las cuatro secciones restantes fueron adaptadas de estudios similares de aceptación de tecnología y evaluación heurística en diferentes casos de estudio (Rauniar et al. 2014; Li et al., 2016; Park et al., 2016; Chintalapati y Daruri, 2017). Los 17 ítems resultantes fueron identificados según el criterio TAM al que responden, tal y como se ilustra en la tabla 2. Estos ítems se midieron utilizando escala de Likert con valores desde "no significativo" (1) hasta "significativo en extremo" (5).

Tabla 2: Ítems de la encuesta relacionados con los criterios de TAM y evaluación heurística.

\begin{tabular}{|l|l|}
\hline Identificador & \\
\hline a1 & Es favorable su actitud hacia el uso del aplicativo informático. \\
\hline a2 & El aplicativo informático se relaciona con las tareas académicas. \\
\hline peou1 & El manejo del aplicativo informático es fácil. \\
\hline peou2 & El usuario, en poco tiempo, puede convertirse en un experto en el uso del aplicativo. \\
\hline peou3 & El diseño del aplicativo informático facilita su uso. \\
\hline peou4 & Todas las funcionalidades del aplicativo son accesibles. \\
\hline peou5 & Para el manejo del aplicativo informático realiza un gran esfuerzo mental. \\
\hline peou6 & El aplicativo informático muestra de manera clara sus funcionalidades. \\
\hline pu1 & El aplicativo informático mejora la comunicación entre los docentes y estudiantes. \\
\hline pu2 & El aplicativo informático es útil en la formación académica. \\
\hline pu3 & Incluiría el uso de este aplicativo informático en todas las materias. \\
\hline pu4 & Este aplicativo informático le permitirá aumentar sus conocimientos. \\
\hline pu5 & Con el uso del aplicativo informático tendrá un mayor interés en las materias. \\
\hline pu6 & El aplicativo informático ayuda a despejar dudas en las tareas académicas. \\
\hline bi1 & De quedar disponible el sistema, lo usaría. \\
\hline bi2 & Tengo intención de utilizar la aplicación para compartir contenido con otros usuarios. \\
\hline bi3 & Tengo intención de utilizar la aplicación para aprender de otros \\
\hline
\end{tabular}

La encuesta fue aplicada en un laboratorio de computación, lo que permitió el uso de Google Forms en condiciones controladas. Durante la aplicación de la encuesta se entregó la información necesaria, se aclararon las dudas y se solucionaron las inquietudes que pudieron surgir, sin influir en la respuesta de los participantes en el estudio. Los datos recogidos fueron procesados estadísticamente con ayuda del IBM SPSS Statistics ver. 23.

En cuanto al tamaño de muestra del experimento, los grupos estuvieron formados por 21 estudiantes cada uno. El $100 \%$ de estudiantes convocados participaron en el experimento siendo estudiantes que cursan tercer o cuarto nivel de la carrera de Ingeniería en Sistemas Computacionales e Informáticos, de la Universidad Técnica de Ambato. Se trabajó con estudiantes de años intermedios teniendo en cuenta que, según Junco (2015), el uso de Facebook no tiene un efecto negativo en el rendimiento de estudiantes de este nivel.

\section{RESULTADOS}

Se logró la implementación y despliegue de la aplicación Facebook Sigma, desarrollada y hospedada en la Universidad Técnica de Ambato. Esta aplicación se integra al canvas de Facebook por lo que, al usarla, la ubicación de la aplicación es transparente al usuario (ver figura 2). Su apariencia es similar, y sus funcionalidades básicas comunes, a las del grupo Facebook con el que se comparó (ver figura 1). Adicionalmente, gestiona la información por categorías e implementa mecanismos de ordenamiento y filtrado. 


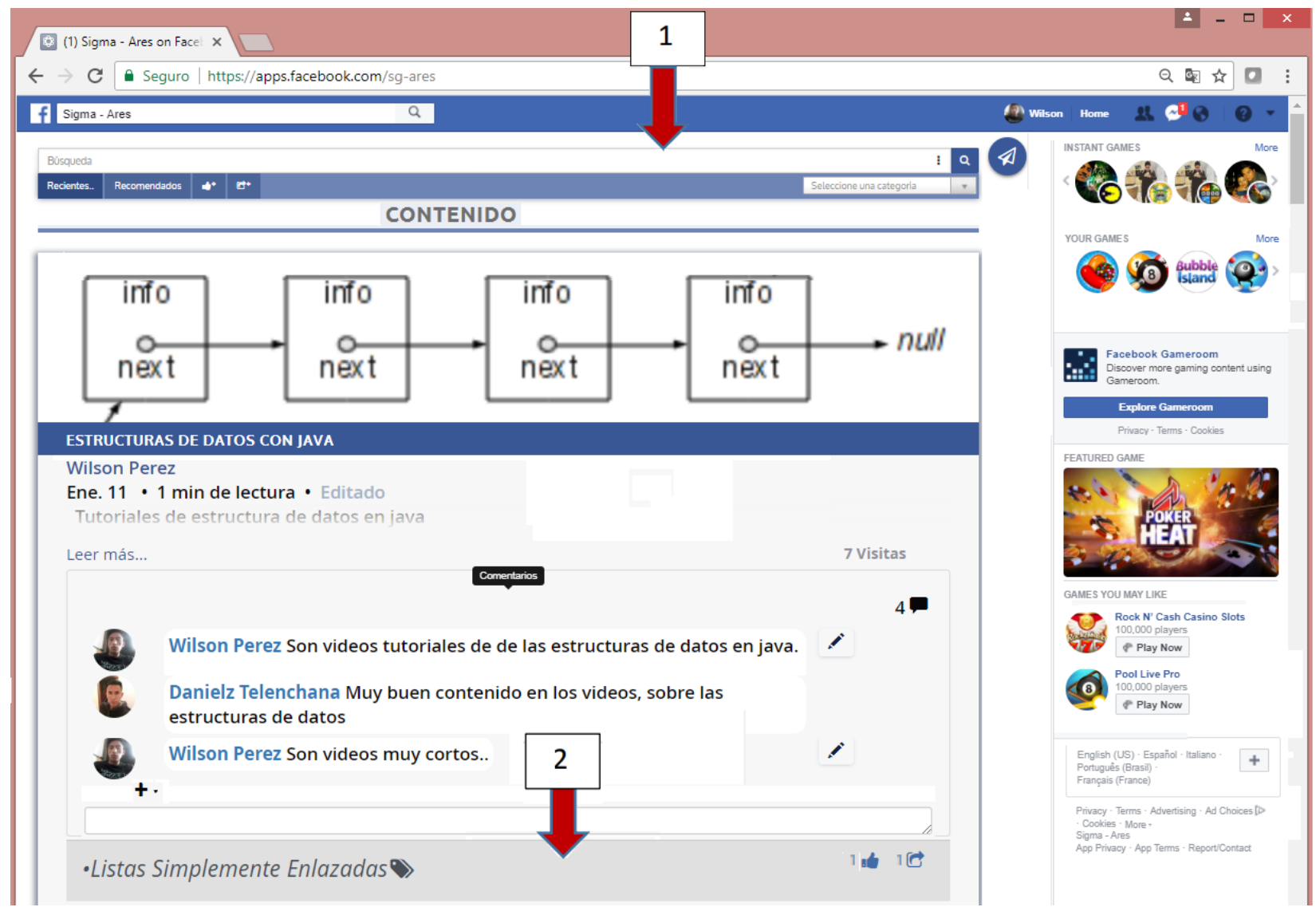

Fig. 2: Apariencia de la aplicación Sigma. Se destaca: 1) barra de búsqueda y filtrado de recursos por diferentes criterios; 2) Barra de acceso rápido a categorías.

La posibilidad de consultar estadísticas del nivel de interacción social alrededor de los recursos compartidos en la propia aplicación Sigma (ver figura 3) es una funcionalidad que ayuda al docente en su labor de facilitador del proceso de aprendizaje para conocer la influencia de la interacción social en el proceso de aprendizaje colaborativo y controlar el proceso de enseñanza.

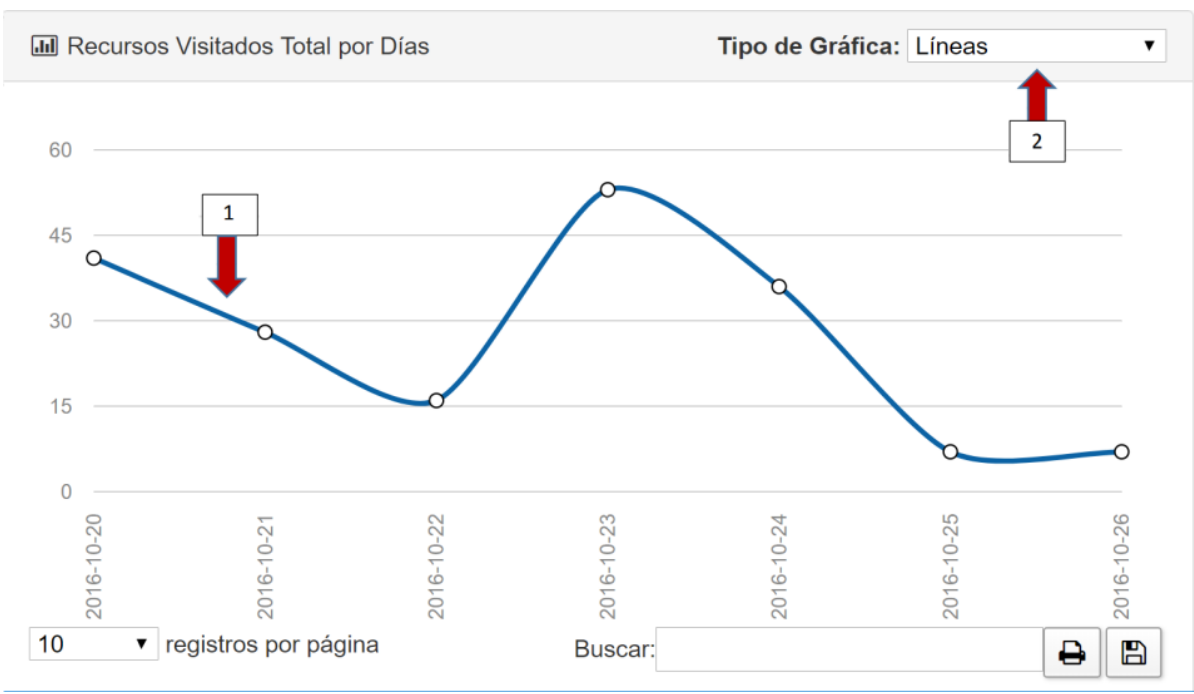

Fig. 3: Ejemplo de estadísticas generadas por Sigma. Se destaca: 1) gráfica ejemplo de la evolución de recursos visitados en un periodo de tiempo dado; 2) posibilidad de seleccionar el tipo de gráfica a generar.

En cuanto al experimento llevado a cabo, como se puede comprobar en la tabla 3 , los estudiantes participantes en el experimento fueron mayoritariamente de sexo masculino y fundamentalmente de dedicación exclusiva al estudio. La edad estuvo entre los 18 y 25 años. Como resultado de la actividad de ambos grupos con Sigma y el grupo Facebook, respectivamente, los estudiantes que utilizaron el grupo Facebook compartieron un $10 \%$ más de recursos y el número de interacciones sociales en la red social a través de esta aplicación fue superior en un $15 \%$. 
Tabla 3: Información demográfica de participantes en el experimento

\begin{tabular}{|l|l|c|c|}
\hline \multicolumn{2}{|c|}{ Información Demográfica } & Sigma (\%) & Grupo Facebook (\%) \\
\hline \multirow{3}{*}{ Sexo } & Femenino & 14 & 19 \\
\cline { 2 - 4 } & Masculino & 86 & 81 \\
\hline \multirow{2}{*}{$\begin{array}{l}\text { Situación } \\
\text { laboral }\end{array}$} & Trabaja & 10 & 29 \\
\cline { 2 - 4 } & No trabaja & 90 & 71 \\
\hline \multirow{2}{*}{ Edad } & $18-20$ años & 43 & 44 \\
\cline { 2 - 4 } & $20-25$ años & 57 & 56 \\
\hline \multirow{2}{*}{ Semestre } & Tercero & 81 & 78 \\
\cline { 2 - 4 } & Cuarto & 19 & 22 \\
\hline
\end{tabular}

A partir de los datos recolectados en la encuesta, se calculó el Alfa de Cronbach de las respuestas a las preguntas de la tabla 2. El valor de este coeficiente fue de 0,927 para la encuesta referente al uso de Sigma, y 0,838 para la encuesta referente al uso del grupo Facebook. Estos resultados permitieron corroborar la validez interna de los datos recolectados. Se procedió entonces a analizar la correlación entre los ítems de la encuesta para lo que se realizó un análisis de componentes principales (PCA, del inglés Principal Component Analysis). Se utilizó normalización Varimax con Kaiser como método de rotación y la rotación convergió en siete iteraciones. Los valores resultantes se muestran en la tabla 4. En la matriz de componentes rotados, para Sigma, las respuestas al cuestionario convergieron en cuatro componentes mientras que, para el grupo de control, lo hicieron en cinco componentes; esto determina la cantidad de columnas de la tabla 4.

Tabla 4: Matriz de componentes rotados del PCA aplicado al grupo experimental y de control.

\begin{tabular}{|c|c|c|c|c|c|c|c|c|c|}
\hline \multirow{2}{*}{$\begin{array}{c}\text { Identificador } \\
\text { de Ítem }\end{array}$} & \multicolumn{4}{|c|}{ Componentes (Sigma) } & \multicolumn{5}{|c|}{ Componentes (Grupo Facebook) } \\
\hline & 1 & 2 & 3 & 4 & 1 & 2 & 3 & 4 & 5 \\
\hline a1 & , 179 & ,134 & 812 & ,455 &,- 062 & , 190 & , 144 & ,925 & ,089 \\
\hline a2 & , 192 & ,122 & ,867 &,- 030 & ,429 & ,031 &,- 194 & 695 & ,436 \\
\hline peou1 & ,386 & ,225 & ,805 & ,042 & 075 & ,215 &,- 049 & ,133 & ,884 \\
\hline peou2 & ,634 & 201 & 176 & ,316 & 151 & 861 &,- 110 &,- 049 & ,251 \\
\hline peou3 & ,591 & 292 & ,431 &,- 340 & ,251 & 832 &,- 073 & ,314 & ,234 \\
\hline peou4 & ,840 & , 182 & ,180 & ,023 &,- 178 & ,781 & ,200 & ,327 & 194 \\
\hline peou5 &,- 850 &,- 082 & ,068 & ,118 & 200 &,- 371 & ,110 &,- 105 &,- 672 \\
\hline peou6 & 850 & 223 & , 180 & 232 & ,178 & ,401 & ,014 & ,509 & ,547 \\
\hline pu1 & ,041 & 826 & 294 & 206 & 206 &,- 056 & 901 & , 110 &,- 053 \\
\hline pu2 & ,004 & 698 & ,368 & , 128 & 259 & ,423 & ,408 & ,549 & 225 \\
\hline pu3 & ,577 & ,735 & ,032 &,- 022 & ,110 & ,071 & 881 & ,043 &,- 094 \\
\hline pu4 & ,489 & 605 & ,095 & ,436 & 643 &,- 178 & ,580 &,- 082 &,- 113 \\
\hline pu5 & ,376 & ,755 & ,064 & 208 & ,834 & , 185 &,- 197 & ,255 &,- 187 \\
\hline pu6 & ,596 & 379 & ,271 & ,418 & ,756 &,- 080 & ,456 &,- 051 & ,193 \\
\hline bi1 & ,377 & ,434 &,- 030 & ,752 & ,577 & ,281 & ,389 & ,480 &,- 142 \\
\hline bi2 & , 110 & ,172 & ,239 & ,895 & ,772 & ,053 & ,331 & ,198 & ,098 \\
\hline bi3 &,- 068 &, 546 & 642 & ,284 & 901 &, 123 & ,125 &,- 011 & ,058 \\
\hline
\end{tabular}

Del análisis de la correlación entre los ítems que formaron grupos homogéneos en ambos aplicativos, se obtuvieron las siguientes ideas, que son obvias para el tipo de aplicativo que se estudió, reforzando así, la validez de la encuesta: 1) el hecho de que el aplicativo se relacione con las tareas académicas (a2) está fuertemente correlacionado con lo favorable que es la actitud de los encuestados hacia el uso de este (a1); 2) el que en poco tiempo se pueda llegar a ser un experto en el manejo del aplicativo (peou2) está fuertemente correlacionado con la medida en que el diseño del aplicativo facilita su manejo (peou3) y la accesibilidad de sus funcionalidades (peou4); 3) al mejorar la comunicación entre docentes y estudiantes (pu1) se sugiere que el aplicativo sea incluido en todas las materias (pu3); 4) al incrementar el interés en las materias a través del 
uso del aplicativo (pu5) aumenta el conocimiento adquirido (pu4); y 5) la intención de usar el sistema (bi1), está fuertemente correlacionada con la intención de compartir contenido con otros usuarios (bi2).

Las diferencias entre los componentes resultantes implican que cuán fácil es el manejo del aplicativo informático (peou1) quedó fuertemente correlacionado con otros criterios de la facilidad de uso percibida para el grupo Facebook, pero no para el caso de Sigma, donde se reflejó una correlación fuerte con la actitud hacia el uso. Del mismo modo, la medida en que el grupo Facebook es útil en la formación académica (pu2) convergió con la actitud hacia el uso (con un valor de apenas 0,549) mientras que, en el caso de Sigma, pu2 converge con otros cuatro ítems que también evalúan usabilidad percibida. Estos resultados dan indicio de que la resistencia al cambio afectó negativamente la opinión de los estudiantes con relación al uso de Sigma.

Por su parte, la medida en que el aplicativo informático ayuda a despejar dudas en las tareas académicas (pu6) converge con otros criterios de usabilidad percibida y de intención de uso, para el grupo de Facebook y, con apenas un valor de 0,596 con ítems de facilidad de uso percibida, para el caso de Sigma. Así mismo, la intención de utilizar la aplicación para aprender de otros (bi3) está muy fuertemente correlacionada con el resto de ítems de intención de uso para el grupo de Facebook y en el caso de Sigma, está correlacionada con ítems de actitud hacia el uso. Estos resultados se interpretan como una evidencia de que los estudiantes podrían estar acostumbrados a consultar a sus compañeros a través de un grupo Facebook para despejar dudas en las tareas académicas y que consideran que esta es una forma válida para aprender de otros.

La tabla 5 muestra los resultados del cálculo de estadísticos descriptivos a los 17 ítems relacionados con los criterios de TAM y evaluación heurística. Se incluye la mediana (M) y rango intercuartil (RIQ) puesto que se trabajó con datos ordinales, y la media $(\bar{x})$ y desviación típica $(S)$ por el interés que el resultado genera en ciertos casos. Aparentemente, el uso del grupo Facebook tuvo mayor aceptación que la aplicación Sigma aunque se requiere un análisis más profundo para concluir al respecto.

Tabla 5: Estadística descriptiva del resultado de la encuesta

\begin{tabular}{|l|c|c|c|c|c|c|c|c|}
\hline \multirow{2}{*}{$\begin{array}{c}\text { Identificador de } \\
\text { Ítem }\end{array}$} & \multicolumn{5}{|c|}{ Sigma } & \multicolumn{4}{c|}{ Grupo Facebook } \\
\cline { 2 - 9 } a1 & $M$ & $R I Q$ & $\bar{x}$ & $S$ & $M$ & $R I Q$ & $\bar{x}$ & $S$ \\
\hline a2 & 4 & 1 & 3,57 &, 811 & 4 & 1 & 4,33 &, 730 \\
\hline peou1 & 4 & 1 & 3,48 &, 928 & 4 & 0 & 4,00 &, 632 \\
\hline peou2 & 4 & 1 & 6,62 & 1,024 & 4 & 1 & 4,14 &, 727 \\
\hline peou3 & 4 & 1 & 3,43 &, 926 & 4 & 1 & 3,90 &, 625 \\
\hline peou4 & 3 & 1 & 3,57 &, 926 & 4 & 0 & 3,95 &, 498 \\
\hline peou5 & 3 & 1 & 3,29 &, 717 & 4 & 1 & 4,00 &, 707 \\
\hline peou6 & 2 & 1 & 2,71 &, 784 & 2 & 1 & 2,24 & 1,179 \\
\hline pu1 & 3 & 1 & 3,38 &, 805 & 4 & 1 & 3,76 &, 625 \\
\hline pu2 & 4 & 1 & 3,57 &, 598 & 4 & 2 & 3,76 & 1,179 \\
\hline pu3 & 4 & 1 & 3,62 &, 669 & 4 & 1 & 4,05 &, 669 \\
\hline pu4 & 3 & 1 & 3,38 &, 669 & 4 & 1 & 3,67 & 1,065 \\
\hline pu5 & 3 & 1 & 3,43 &, 598 & 4 & 1 & 3,48 & 1,123 \\
\hline pu6 & 3 & 1 & 3,48 &, 680 & 3 & 1 & 3,43 &, 926 \\
\hline bi1 & 3 & 1 & 3,48 &, 680 & 4 & 1 & 3,48 & 1,078 \\
\hline bi2 & 3 & 1 & 3,57 &, 811 & 4 & 1 & 3,81 &, 602 \\
\hline bi3 & 4 & 2 & 3,76 &, 831 & 4 & 1 & 3,71 &, 845 \\
\hline
\end{tabular}

Para determinar el nivel de significancia de las diferencias entre las respuestas del grupo que utilizó Sigma y el que utilizó el grupo Facebook, se llevó a cabo un análisis de normalidad utilizando la prueba de Shapiro-Wilk $(n=21)$, corroborando que los valores asignados a los ítems de la referida encuesta no siguen una distribución normal. Por esta razón, se optó por llevar a cabo una prueba no paramétrica de dos muestras independientes. Los resultados permitieron validar que la diferencia es significativa con un valor $p$ menor a ,05 para los casos de cuán favorable es la actitud del encuestado hacia el uso del aplicativo (a1), en qué medida el aplicativo se relaciona con las tareas académicas (a2), la accesibilidad de las funcionalidades del aplicativo (peou4) y la utilidad del aplicativo en la formación académica (pu2), tal y como se refleja en la tabla 6. En estos ítems, la respuesta fue favorable para el uso del grupo Facebook por lo que se rechaza la hipótesis nula. En la tabla 6 no se muestran el resto de valores puesto que el valor $p$ no fue menor de ,05. 
Tabla 6: Resultados significativos de la prueba no paramétrica de dos muestras independientes.

\section{DISCUSIÓN}

\begin{tabular}{|l|l|l|l|l|}
\hline & \multicolumn{1}{|c|}{ a1 } & \multicolumn{1}{c|}{ a2 } & peou4 & \multicolumn{1}{c|}{ pu2 } \\
\hline U de Mann-Whitney & 112,500 & 148,500 & 117,000 & 147,000 \\
\hline W de Wilcoxon & 343,500 & 379,500 & 348,000 & 378,000 \\
\hline Z & $-2,888$ & $-1,987$ & $-2,818$ & $-2,025$ \\
\hline Sig. Asintótica (bilateral) &, 004 &, 047 &, 005 &, 043 \\
\hline
\end{tabular}

Con relación al desarrollo de Sigma, es importante destacar que fue posible agregar las funcionalidades nuevas en Sigma, con relación a lo que ofrece un grupo Facebook, gracias a que se integró en el canvas de Facebook una aplicación que maneja su propia base de datos, en la que se almacena la información gestionada, incluyendo las interacciones sociales que también son registradas en Facebook. Al ordenar la información por categorías de interés, se busca disminuir la sobrecarga de información; con los mecanismos de ordenamiento y filtrado se pretende facilitar el acceso a información compartida de mayor impacto. Se podría entender que la existencia de usuarios que jueguen el rol de quienes no hacen compartir o me gusta sobre los recursos de información publicados impide que los participantes en el aprendizaje colaborativo cuenten con las mismas oportunidades. Sin embargo, consideramos que la creación de este rol permite que, a diferencia de con un grupo Facebook, la aplicación Sigma se pueda utilizar aun cuando ciertos estudiantes y/o facilitadores del proceso de aprendizaje colaborativo no tengan usuario en Facebook y no deseen tenerlo.

Respecto al método de evaluación de resultados, en los trabajos revisados se destaca como ventajas, tanto de evaluación heurística como de TAM, la facilidad de recolección de datos, la pertinencia de los resultados y la sencillez de la metodología de evaluación. A partir del estudio de métodos de evaluación llevado a cabo, fue que se decidió utilizar evaluación heurística y TAM para evaluar las herramientas en estudio. Pimmer et al. (2017) y Rap y Blonder (2017) sentaron un precedente para esta investigación, al haber estudiado el uso de Facebook como herramienta para supervisar el componente de investigación en equipos de estudiantes participantes en un proceso de aprendizaje colaborativo. Sin embargo, el trabajo de Pimmer et al. se limitó a un análisis cualitativo basado en entrevistas y el de Rap y Blonder se enfoca únicamente en el análisis de la evolución del rendimiento académico durante el experimento. En las estadísticas descriptivas, se aprecia que los estudiantes que evaluaron Sigma reportaron una intención de uso del aplicativo en el ámbito educativo mayor que la de los estudiantes que evaluaron el uso de los grupos Facebook. Esta idea es lógica si tenemos en cuenta que Sigma fue diseñado para su uso en el contexto académico. Sin embargo, este resultado no es estadísticamente significativo.

En comparación con los resultados de otros investigadores, Li et al (2018) defienden la flexibilidad de la plataforma Facebook en las más diversas áreas del conocimiento; pero en la literatura consultada no se encontraron otras propuestas de una aplicación que personaliza el uso de la red social Facebook en el ámbito académico, como lo hace Sigma. No obstante, y a pesar de las preocupaciones de Dohn (2017) referentes a las barreras en la comunicación que se pueden generar en el uso de grupos Facebook, el uso de Sigma no demostró ser una mejor alternativa. Los resultados están en consonancia con la pertinencia del uso de grupos Facebook en el ámbito académico comprobada experimentalmente por Castro y González-Palta (2016), Chang et al. (2017), Dohn (2017) y Guo et al. (2018) pero en el contexto del uso de esta tecnología para la formación universitaria de estudiantes de Ingeniería en Sistemas.

\section{CONCLUSIONES}

Con el desarrollo del presente trabajo se concluye que: 1) la flexibilidad de la plataforma Facebook permitió la construcción de una aplicación que personaliza el uso de esta red social en el ámbito académico y, contrario a lo supuesto, 2) la aceptación de la tecnología de la plataforma Facebook utilizando un grupo Facebook es superior a la del uso de la aplicación Facebook construida, aun cuando esta fue diseñada específicamente para apoyar el proceso de aprendizaje. Los grupos Facebook se mantienen como la alternativa que garantiza la mayor aceptación de la tecnología de la plataforma Facebook en el ámbito académico para el aprendizaje colaborativo de estudiantes de Ingeniería en Sistemas de la Universidad Técnica de Ambato.

\section{REFERENCIAS}

Castro, P. e I. González-Palta, Percepción de Estudiantes de Psicología sobre el Uso de Facebook para Desarrollar Pensamiento Crítico, doi: 10.4067/S0718-50062016000100006, Formación Universitaria, 9(1), 45-56 (2016)

Chang, L., J. Guo y H. Lin, Cultural Competence Education for Health Professionals from Pre-graduation to Licensure Delivered using Facebook: Twelve-month follow-up on a Randomized Control Trial, doi: 10.1016/j.nedt.2017.09.005, Nurse Education Today, 59, 94-100 (2017) 
Chintalapati, N. y V. Daruri, Examining the Use of YouTube as a Learning Resource in Higher Education: Scale Development and Validation of TAM Model, doi: 10.1016/j.tele.2016.08.008, Telematics and Informatics, 34(6), 853-860 (2017)

Çoklar, A., Evaluations of Students on Facebook as an Educational Environment, ISSN: 1309-6591, Turkish Online Journal of Qualitative Inquiry, 3(2), $42-53$ (2012)

De La Hoz, L. P., D. Acevedo y J. Torres, Uso de Redes Sociales en el Proceso de Enseñanza y Aprendizaje por los Estudiantes y Profesores de la Universidad Antonio Nariño, Sede Cartagena, doi: 10.4067/S0718-50062015000400009, Formación Universitaria, 8(4), 77-84 (2015)

Dohn, N.B., Integrating Facebook in Upper Secondary Biology Instruction: A Case Study of Students' Situational Interest and Participation in Learning Communication, doi: 10.1007/s11165-016-9549-3, Research in Science Education, 47(6), 1305-1329 (2017)

Guo, R., Y. Shen y L. Li, Using Social Media to Improve Student-Instructor Communication in an Online Learning Environment, doi: 10.4018/IJICTE.2018010103, International Journal of Information and Communication Technology Education, 14(1), 33-43 (2018)

Hammouri, Q. y E. Abu-Shanab, Exploring Factors afecting Users' satisfaction toward E-learning Systems, doi: 10.4018/IJICTE.2018010104, International Journal of Information and Communication Technology Education, 14(1), 44-57 (2018)

Junco, R., Student Class Standing, Facebook Use, and Academic Performance, doi:10.1016/j.appdev.2014.11.001, Journal of Applied Developmental Psychology, 36, 18-29 (2015)

Kirschner, P., Facebook as Learning Platform: Argumentation Superhighway or Dead-End Street? doi: 10.1016/j.chb.2015.03.011, Computers in Human Behavior, 53, 621-625 (2015)

Lagunes, A., C. Torres, J. Angulo y M. Martínez, Prospectiva hacia el Aprendizaje Móvil en Estudiantes Universitarios, doi: 10.4067/S0718-50062017000100011, Formación Universitaria, 10(1), 101-108 (2017)

Li, S., Q. Guo, K. Yang, J. Liu e Y. Zhang, Uncovering the Popularity Mechanisms for Facebook Applications, doi: 10.1016/j.physa.2017.12.006, Physica A: Statistical Mechanics and its Applications, 494, 422-429 (2018)

Li, T., M. Chau, W. Sung, A. Lee, P. Wong y P. Yip, Design and Evaluation of a Facebook Game for Self-Directed ELearning, Knowledge Management and E-Learning, 8(3), 464-480 (2016)

Lin, G., Annonymous versus Identified Peer Assessment via a Facebook-based Learning Application: Effects on Quality of Peer Feedback, Perceived Learning, Perceived Fairness, and Attitude toward the System, doi: 10.1016/j.compedu.2017.08.010, Computers and Education, 116, 81-92 (2018)

Manca, S. y M. Ranieri, Is it a Tool Suitable for Learning? A Critical Review of the Literature on Facebook as a Technologyenhanced Learning Environment, doi: 10.1111/jcal.12007, Journal of Computer Assisted Learning, 29(6), 487-504 (2013)

McCarthy, J., Learning in the Café: Pilot testing the Collaborative Application for Education in Facebook, doi: 10.14742/ajet.1500, Australasian Journal of Educational Technology, 31(1), 67-85 (2015)

Moghavvemi, S., T. Paramanathan, N. Rahin y M. Sharabati, Student's perceptions towards using E-Learning via Facebook, doi: 10.1080/0144929X.2017.1347201, Behaviour and Information Technology, 36(10), 1081-1100 (2017)

Park, B.K., E. Nahm y V. Rogers, Development of a Teen-Friendly Health Education Program on Facebook: Lessons Learned, doi: 10.1016/j.pedhc.2015.06.011, Journal of Pediatric Health Care, 30(3), 197-207 (2016)

Pimmer, C., J. Chipps y otros cuatro autores, Facebook for supervision? Research Education shaped by the Structural Properties of a Social Media Space, doi: 10.1080/1475939X.2016.1262788, Technology, Pedagogy and Education, 26(5), 517-528 (2017)

Po-Hsun, Ch. y Ch. Li-Wei, Peer Learning Efficacy Analysis on Undergraduate Software Design Course, doi: 10.1002/cae.21856, Computer Applications in Engineering Education, 26(1), 5-16 (2018)

Rap, S. y R. Blonder, Thou, Shall not Try to speak in the Facebook Language: Students' perspectives regarding using Facebook for Chemistry Learning, doi: 10.1016/j.compedu.2017.06.014, Computers and Education, 114, 69-78 (2017)

Rauniar, R., G. Rawski, J. Yang y B. Johnson, Technology Acceptance Model (TAM) and Social Media Usage: an Empirical Study on Facebook, doi: 10.1108/JEIM-04-2012-0011, Journal of Enterprise Information Management, 27(1), 6-30 (2014)

Scherer, R., J. Tondeur, F. Siddiq y E. Baran, The importance of Attitudes toward Technology for Pre-Service Teachers' Technological, Pedagogical, and Content Knowledge: Comparing Structural Equation Modelling Approaches, doi: 10.1016/j.chb.2017.11.003, Computers in Human Behavior, 80, 67-80 (2018)

Thompson, P., Communication Technology Use and Study Skills, doi: 10.1177/1469787417715204, Active Learning in Higher Education, 18(3), 257-270 (2017)

Tsovaltzi, D., R. Judele, T. Puhl y A. Weinberger, Leveraging Social Networking Sites for Knowledge Construction: Positive Effects of Argumentation Structure, but Premature Knowledge Consolidation after Individual Preparation, doi: 10.1016/j.learninstruc.2017.06.004, Learning and Instruction, 52, 161-179 (2017)

Zorkociova, O. y L. Vankova, The Phenomenon of Social Networks and the Effectiveness of Facebook Page Measuring (the case of Slovakia), doi: 10.21003/ea.V160-16, Economic Annals-XXI, 160(7-8), 83-87 (2016) 